\title{
Influence of the Friction Coefficient on the Trajectory Performance for a Car-Like Robot
}

\author{
Francisco Valero, ${ }^{1}$ Francisco Rubio, ${ }^{1}$ Carlos Llopis-Albert, ${ }^{2}$ and Juan Ignacio Cuadrado ${ }^{1}$ \\ ${ }^{1}$ Center of Technological Research in Mechanical Engineering, Universitat Politècnica de València, Building 5E, Valencia, Spain \\ ${ }^{2}$ Department of Mechanical Engineering and M. Strength, Universitat Politècnica de València, Valencia, Spain
}

Correspondence should be addressed to Francisco Rubio; frubio@mcm.upv.es

Received 30 March 2017; Accepted 24 May 2017; Published 22 June 2017

Academic Editor: Rafael Morales

Copyright (C) 2017 Francisco Valero et al. This is an open access article distributed under the Creative Commons Attribution License, which permits unrestricted use, distribution, and reproduction in any medium, provided the original work is properly cited.

\begin{abstract}
A collision-free trajectory planner for a car-like mobile robot moving in complex environments is introduced and the influence of the coefficient of friction on important working parameters is analyzed. The proposed planner takes into account not only the dynamic capabilities of the robot but also the behaviour of the tire. This planner is based on sequential quadratic programming algorithms and the normalized time method. Different values for the coefficient of friction have been taken following a normal Gaussian distribution to see its influence on the working parameters. The algorithm has been applied to several examples and the results show that computation times are compatible with real-time work, so the authors call them efficient generated trajectories as they avoid collisions. Besides, working parameters such as the minimum trajectory time, the maximum vehicle speed, computational time, and consumed energy have been monitored and some conclusions have been reached.
\end{abstract}

\section{Introduction}

Trajectory planning in Robotics has been a field of active work for decades, and, in recent years, its application to mobile robots and autonomous vehicles has gained particular relevance, given the importance of industrial use in the case of robots and safety in the area of vehicles $[1,2]$.

These uses introduce the need for nonholonomic restrictions when the robot is modelled, and in many cases this raises the issue of obtaining the trajectory as an optimization problem, as in Peng et al. [3]. In the case of autonomous vehicles, there are specific problems associated with driving on different road types (see Katrakazas et al. [4]).

Many authors have based the generation of trajectories on paths adjusted for different functions using, for example, Bezier curves [5, 6], B-splines, as in Elbanhawi et al. [7], clothoid curves (see Broggi et al. [8]), and polynomials $[9,10]$.

Techniques for trajectory planning seek continuous functions and their derivatives, with the consequent impact on the robot's kinematic and dynamic response, which will also be subject to specific restrictions in each case.
Effectiveness and operating capability of performing a prescribed trajectory for mobile robots, as for any other vehicle, will depend heavily on their dynamic behaviour.

In cases where the trajectory is generated along a previously obtained path without considering the dynamic constraints of the robot and its ability to transmit forces to the ground, its feasibility is not guaranteed, nor is the time required for it to be performed, so the efficiency of work done by the robot cannot be verified.

Significant simplifications are required to model the dynamics of the robot so that it can work with reduced computing times, and in four-wheel (car-type) robots it is usual to work with the "bicycle" model and different alternatives when considering tire behaviour, from neglecting them, as in Wang and Qi [11], to different approaches, as in Jeon et al. [12] and Cong et al. [13]. See also the dynamic model proposed by Staicu [14].

This paper presents a planner for obtaining trajectories for four-wheel robots while considering their dynamic properties. This planner, which is able to work efficiently, is based on solving optimization problems recursively. 
The values of the coefficient of friction have been taken from a normal Gaussian distribution.

One objective of this paper is to determine the influence of the coefficient of friction on important operating parameters such as minimum trajectory time, maximum speed of the robot, computational time, and consumed energy.

The dynamic approach includes modelling the entire carlike robot, including tires, engine, brakes, and transmission system, with the aim of seeking feasible and efficient trajectories for the robot and analysing the influence of the coefficient of friction on the working parameters. This approach marks a clear difference with other planners that only include kinematic constraints, as in Simba et al. $[15,16]$. Those planners either are conservative or do not guarantee the feasibility of the trajectories, as in Li and Shao [17] and Tokekar et al. [18].

Efficient computation time is achieved by simplifying the dynamic model, rapidly evaluating collisions, as explained in Rubio et al. [19], and generating a trajectory based on a path that is composed of parts which are defined by coordinates corresponding to polynomial functions of time. The efficiency of the trajectory is due to the fact that it uses the full dynamic capability of the robot. It starts from an initial minimum time trajectory that is adapted to environmental obstacles, the efficiency of which is shown in the examples analysed. A lot of examples (up to 100) have been solved by varying the coefficient of friction. The results have been monitored using the corresponding graphs in Section 5.

\section{Dynamic Model of the Robotic Vehicle}

In this section, we present the modelling of the dynamic behaviour of a mobile robot with tires. This model will be used in an iterative process that requires a sequence of optimization problems to be solved. Dynamic restrictions are essential to find feasible trajectories and are based on simplified but safe modelling.

Other authors have also used a simplified dynamic model, demonstrating its efficiency. For example, in Cazalilla et al. [20], the authors reduce the number of relevant dynamic parameters for the sake of simplicity of the model. Another example of simplified dynamic model can be found in Iriarte et al. [21]. To work in real time, Pastorino et al. [22] also resort to simplification in the modelling of the vehicle.

The robot used as an example has four wheels arranged symmetrically about its central axis, with the driving torque acting on the rear wheels, with braking on all wheels, and with front-wheel steering. The tire behaviour is crucial for determining the dynamic performance of the robot.

In addition, the following simplifying assumptions are considered:

(i) There are no roll and pitch motions.

(ii) There is no side load transfer.

(iii) There are no aerodynamic effects.

(iv) A "bicycle" type, planar model is used with three degrees of freedom and a restriction associated with the steering angle. The front wheels are simplified and
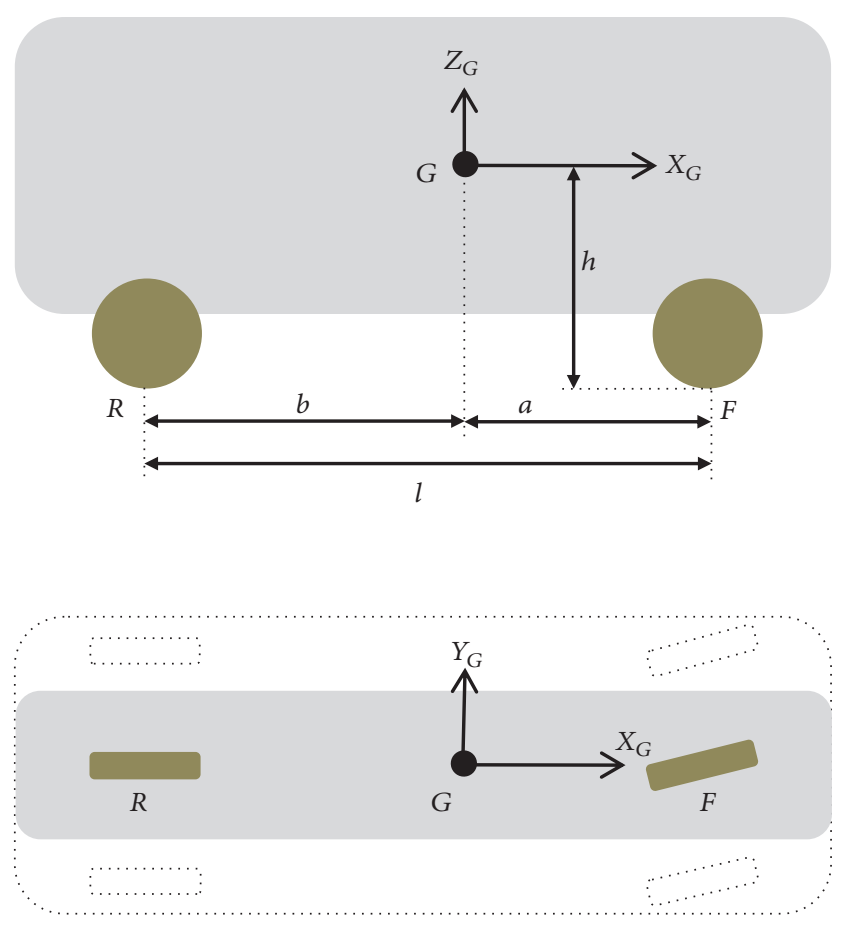

FIGURE 1: Geometry and its simplification.

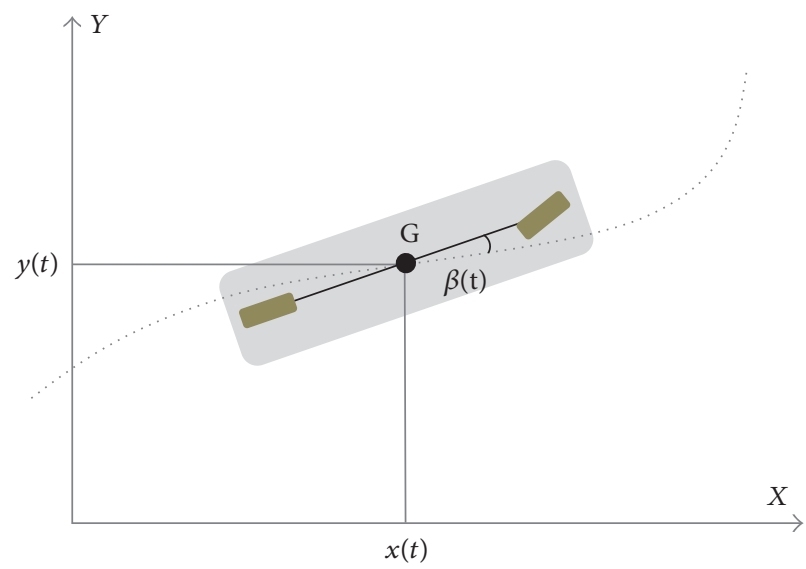

Figure 2: Robot configuration.

replaced by one that will account for the force exerted by the two. The same simplification applies to the rear wheels.

(v) The steering angle is equal for each front wheel and corresponds to the steering angle of the "bicycle model."

The geometry of the robot is shown at the top of Figure 1, in a side view, and the reduction to the "bicycle model" can be seen in the plan view.

2.1. Features of the Trajectory. A trajectory is established using time-dependent Cartesian components of the position (see Figure 2). 


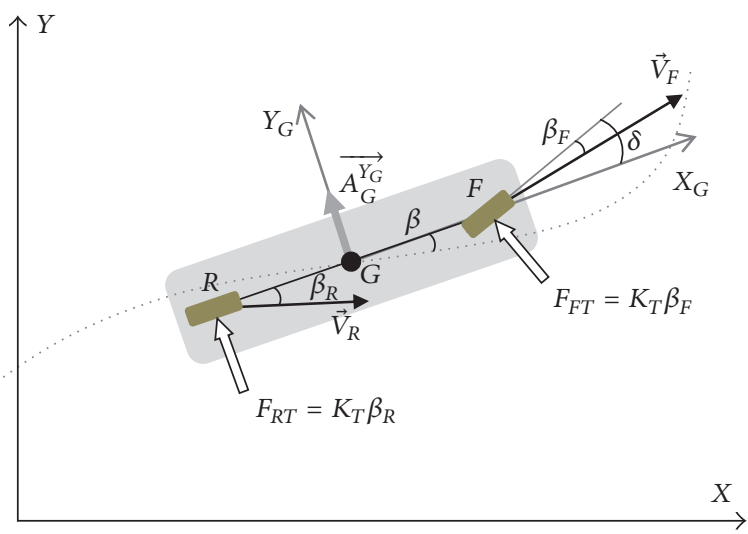

FIGURE 3: Robot kinematics.

The position $(G)$ of the COG of the robot is

$$
\begin{aligned}
& x=a_{x}+b_{x} t+c_{x} t^{2}+d_{x} t^{3}, \\
& y=a_{y}+b_{y} t+c_{y} t^{2}+d_{y} t^{3} .
\end{aligned}
$$

By differentiating with respect to time, the COG's velocity and acceleration are obtained.

The orientation of the velocity of $G$ is

$$
\varphi(t)=\tan ^{-1}\left(\frac{\dot{y}}{\dot{x}}\right)
$$

Considering the small sideslip angles both in the vehicle and in the tires and $\dot{\beta}$ negligible compared to $\dot{\varphi}$, the robot kinematics can be written as a function of (1) and its derivatives.

The sideslip angle in the rear wheel is obtained from the components of its velocity in local coordinates $X_{G} Y_{G}$ (see Figure 3):

$$
\begin{aligned}
\overrightarrow{V_{R}^{l}} \approx & \left(\dot{x}^{2}+\dot{y}^{2}\right)^{1 / 2} \vec{i}_{l} \\
& +\left(\left(\dot{x}^{2}+\dot{y}^{2}\right)^{1 / 2} \beta-b \frac{\dot{x} \ddot{y}-\dot{y} \ddot{x}}{\dot{x}^{2}+\dot{y}^{2}}\right) \vec{j}_{l},
\end{aligned}
$$

where

$$
\beta_{R} \approx \beta-\frac{(\dot{x} \ddot{y}-\dot{y} \ddot{x}) b}{\left(\dot{x}^{2}+\dot{y}^{2}\right)^{3 / 2}}
$$

Proceeding in a similar way to that used to obtain (4), the sideslip angle in the front axle is

$$
\beta_{F} \approx \delta-\beta-\frac{(\dot{x} \ddot{y}-\dot{y} \ddot{x}) a}{\left(\dot{x}^{2}+\dot{y}^{2}\right)^{3 / 2}}
$$

where $\delta$ is the steering angle.

Lateral acceleration in local coordinates $\left(Y_{G}\right)$ direction is

$$
A_{G}^{Y_{G}} \approx(-\ddot{x} \sin \varphi+\ddot{y} \cos \varphi)-(\ddot{x} \cos \varphi+\ddot{y} \sin \varphi) \beta .
$$

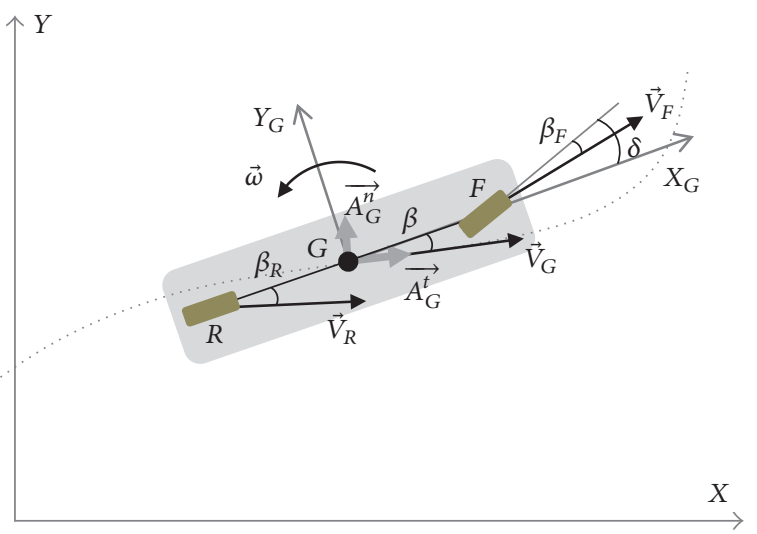

FIGURE 4: Lateral forces on tires.

Under the small sideslip hypothesis, it is usual to consider the lateral behaviour of the tires linearly, so as the front and rear tires are equal, the lateral forces are

$$
\begin{aligned}
& F_{R T}=-K_{T} \beta_{R} \\
& F_{F T}=-K_{T} \beta_{F}
\end{aligned}
$$

with a direction normal to the rim and opposite to the sideslip (see Figure 4).

The equilibrium equation of the side forces of the robot considering small $\delta$ is

$$
F_{R T}+F_{F T}=m A_{G}^{Y_{G}} .
$$

The equation of moments is

$$
a F_{F T}-b F_{R T}=I_{z} \dot{\omega},
$$

where $I_{z}$ is the moment of inertia of the vehicle around an axis parallel to $Z$ passing through $G$.

Equations (8) and (9) are a linear system that enables $\beta$ and $\delta$ to be obtained as functions of the kinematics, tire's lateral stiffness, and the inertial characteristics of the vehicle. Using these values, $\beta_{R}$ and $\beta_{F}$ can be obtained from (4) and (5), respectively.

2.2. Forces Transmitted by the Tire. A force at the contact point of the tire with the ground is considered, so, in local coordinates,

$$
\begin{aligned}
& \vec{F}_{F}=F_{F}^{X_{G}} \vec{i}_{l}+F_{F}^{Y_{G}} \vec{j}_{l}+F_{F}^{Z_{G}} \vec{k}_{l}, \\
& \vec{F}_{R}=F_{R}^{X_{G} \vec{i}_{l}}+F_{R}^{Y_{G}} \vec{j}_{l}+F_{R}^{Z_{G}} \vec{k}_{l} .
\end{aligned}
$$

In $X_{G}$ direction, assuming a small steering angle, the following equilibrium equation is set:

$$
F_{F}^{X_{G}}+F_{R}^{X_{G}}=m A^{X_{G}},
$$

where

$$
A^{X_{G}}=\ddot{x} \cos (\beta+\varphi)+\ddot{y} \sin (\beta+\varphi) .
$$


In the front wheel,

$$
\begin{aligned}
& A^{X_{G}}>0 \longrightarrow \\
& F_{F}^{X_{G}}=0, \\
& A^{X_{G}} \leq 0 \longrightarrow \\
& F_{F}^{X_{G}}=0.6 m A^{X_{G}}+F_{F_{r}}^{X_{G}}
\end{aligned}
$$

with losses due to rolling motion:

$$
F_{F_{r}}^{X_{G}}=\mu_{r} F_{F}^{Z_{G}}
$$

where $\mu_{r}$ is considered constant because the velocity and sideslip angles are small.

The force on the rear wheel is

$$
\begin{aligned}
& A^{X_{G}}>0 \longrightarrow \\
& F_{R}^{X_{G}}=m A^{X_{G}}+F_{R_{r}}^{X_{G}}+F_{F_{r}}^{X_{G}}, \\
& A^{X_{G}} \leq 0 \longrightarrow \\
& F_{R}^{X_{G}}=0.4 m A^{X_{G}}+F_{R_{r}}^{X_{G}}
\end{aligned}
$$

with

$$
F_{R_{r}}^{X_{G}}=\mu_{r} F_{R}^{Z_{G}}
$$

Equations (13) and (15) are in line with the torque applied to the rear wheels (for positive $A^{X_{G}}$ ), while the braking torque is divided between the front (60\%) and rear wheels (40\%) when the acceleration is negative.

Components in direction $Y_{G}$, for small sideslip angles, correspond to a linear lateral slip behaviour of the tire and are given by (7).

Components in direction $Z_{G}$, taking into account only the load transfer due to longitudinal acceleration (direction $X_{G}$ ), are

$$
\begin{aligned}
& F_{F}^{Z_{G}}=\frac{m}{l}\left(b g-A^{X_{G}} h\right), \\
& F_{R}^{Z_{G}}=\frac{m}{l}\left(a g+A^{X_{G}} h\right) .
\end{aligned}
$$

A circle is set, limiting the friction force for the combined forces at each wheel, which prevents them from working with higher friction forces than those defined by $\mu_{t}$.

$$
\begin{aligned}
& \sqrt{\left(F_{F}^{X_{G}}\right)^{2}+\left(F_{F}^{Y_{G}}\right)^{2}}<\mu_{t} F_{F}^{Z_{G}}, \\
& \sqrt{\left(F_{R}^{X_{G}}\right)^{2}+\left(F_{R}^{Y_{G}}\right)^{2}}<\mu_{t} F_{R}^{Z_{G}} .
\end{aligned}
$$

2.3. Driving Force. The car-like mobile robot has an electric engine with rear wheel driving and steering on the front wheels. The maximum torque that can be transmitted to the rear wheels is conditioned by the characteristics of the transmission and the limitations of the engine, so a driving force is achieved which is limited by the curve in Figure 5.

Using this restriction, both the integrity of the transmission and the possibility of achieving the best performance required of the engine transmission system are guaranteed.

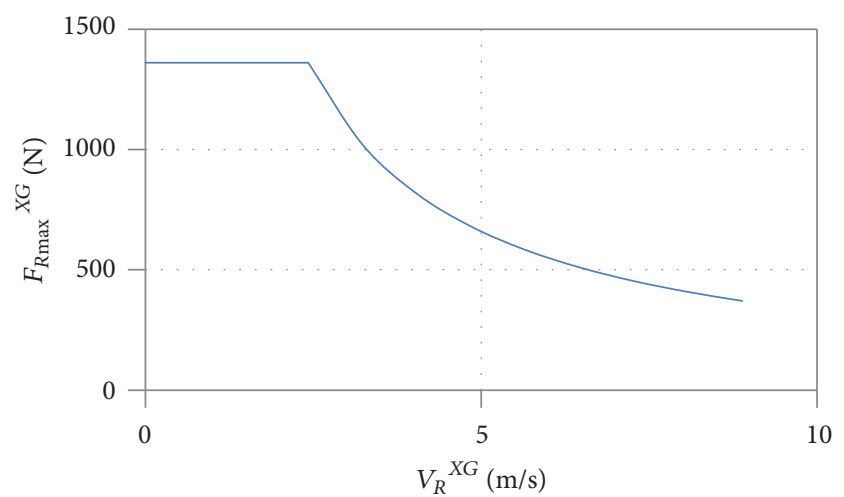

FIGURE 5: Driving force versus velocity.

\section{Modelling of the Trajectory}

The trajectory equations between successive configurations are

$$
\begin{gathered}
\forall t \in\left[0, t_{j}\right] \\
\downarrow \\
x_{j}=a_{x j}+b_{x j} t+d_{x j} t^{2}+e_{x j} t^{3} \\
y_{j}=a_{y j}+b_{y j} t+d_{y j} t^{2}+e_{y j} t^{3},
\end{gathered}
$$

where $j=1, \ldots, m-1$.

A robot configuration is given by the position and orientation of the center of gravity of the mobile robot in global coordinates.

The following conditions associated with the given configurations are considered in order to ensure continuity.

(i) Position. For each interval, the initial and final configurations are known, so $(4(m-1))$ equations are established:

$$
\begin{gathered}
x_{j}(0)=x_{j}, \\
y_{j}(0)=y_{j}, \\
x_{j}\left(t_{j}\right)=x_{j+1}, \\
y_{j}\left(t_{j}\right)=y_{j+1} .
\end{gathered}
$$

(ii) Velocity. The initial and final velocities of the trajectory must be zero, so four new equations are established:

$$
\begin{aligned}
\dot{x}_{1}(0) & =0, \\
\dot{y}_{1}(0) & =0, \\
\dot{x}_{m-1}\left(t_{m}\right) & =0, \\
\dot{y}_{m-1}\left(t_{m}\right) & =0 .
\end{aligned}
$$


At each intermediate step, the final velocity of the previous interval must be equal to the start of the next, leading to $(2(m-2))$ equations:

$$
\begin{aligned}
& \dot{x}_{j}(0)=\dot{x}_{j-1}\left(t_{j-1}\right), \\
& \dot{y}_{j}(0)=\dot{y}_{j-1}\left(t_{j-1}\right) .
\end{aligned}
$$

(iii) Acceleration. At each intermediate step, the final acceleration of the previous interval must be equal to the start of the next, leading to $(2(m-2))$ equations:

$$
\begin{aligned}
& \ddot{x}_{j}(0)=\ddot{x}_{j-1}\left(t_{j-1}\right), \\
& \ddot{y}_{j}(0)=\ddot{y}_{j-1}\left(t_{j-1}\right) .
\end{aligned}
$$

Once the times needed to perform the trajectory between the different configurations for each interval are known, a linear system of equations is established, which makes it possible to obtain the coefficients of the cubic polynomials, providing an efficient solution using the normalized time method (see [23]).

3.1. Minimum Time Trajectory. The minimum time trajectory must also meet the following restrictions:

(i) The driving force cannot exceed the value determined by the limit transmitted to the wheel according to Figure 5:

$$
F_{R}^{X_{G}}(t) \leq F_{R \max }^{X_{G}} \quad \forall t \in\left[0, t_{j}\right] \text { with } j=1, \ldots, m-1 .
$$

(ii) Forces on the tires must not exceed the limit of friction force at any point of the trajectory, so that, according to (18), the following equations are fulfilled:

$$
\begin{aligned}
& \sqrt{\left(F_{F}^{X_{G}}(t)\right)^{2}+\left(F_{F}^{Y_{G}}(t)\right)^{2}}<\mu_{t} F_{F}^{Z_{G}}(t) \\
& \sqrt{\left(F_{R}^{X_{G}}(t)\right)^{2}+\left(F_{R}^{Y_{G}}(t)\right)^{2}}<\mu_{t} F_{R}^{Z_{G}}(t) \\
& \forall t \in\left[0, t_{j}\right] \text { with } j=1, \ldots, m-1 .
\end{aligned}
$$

(iii) The maximum velocity at any point of the trajectory is limited:

$$
\left|\vec{V}_{G}(t)\right|<V_{\max } ; \quad \forall t \in\left[0, t_{j}\right] \text { with } j=1, \ldots, m-1,
$$

where $\vec{V}_{G}(t)$ is the velocity of the center of gravity in global coordinates.

The minimum time is obtained by solving an optimization problem using the restrictions detailed above as variables of time for each interval of the trajectory, and the objective function is as follows:

$$
\sum_{j=1}^{m-1} t_{j}=t_{\min } .
$$

3.2. Obstacles. Patterned obstacles such as spheres, cylinders, and rectangular prisms are used (see [19]), so any geometry will be wrapped up by combinations of these basic obstacles.

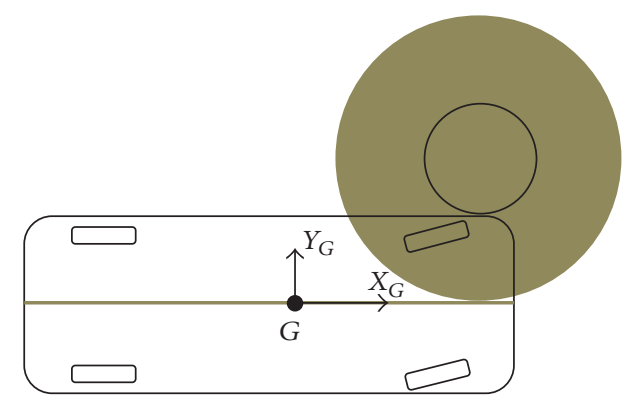

FIGURE 6: Wire-frame vehicle with grown obstacle.

3.3. Collision Detection. The wire-frame model of the robot with grown obstacle techniques (Figure 6) is used to check for collisions (see [24]).

3.4. Generation of the Trajectory without Collision. The problem posed is that of obtaining a feasible and efficient trajectory for a robot in an environment with static obstacles, which enables motion between two given configurations $\left(c_{i}\right.$ and $c_{f}$ ). An efficient trajectory is one which is close to the minimum time with a relatively low computational cost and respects the limitations of the robot dynamics. Obviously, the feasibility of the trajectory implies the absence of collisions.

The process proposed to solve the problem includes the following steps.

3.4.1. Calculation of the Initial Minimum Time Trajectory. Trajectory $T_{\text {min }}$, linked to the sequence of configurations $C=\left\{c_{i}, c_{f}\right\}$, is obtained using the procedure described in Section 3.1.

3.4.2. Search for Collisions. The first configuration from $T_{\min }$ which presents a collision $c_{c}$ is identified, and then a previous and near configuration $c_{a}$ is sought.

3.4.3. Generation of Adjacent Trajectories. Four configurations around $c_{a}$ are generated $\left(c_{a j}, j=1, \ldots, 4\right)$.

3.4.4. Generation of Offspring Trajectories. For each collisionfree $c_{a j}$ configuration obtained in the previous section, the offspring trajectory $T_{k}$ is calculated and is added to the set of $C T$ trajectories, where they are stored and ordered from smaller to longer time.

3.4.5. Trajectory Selection. Then, the minimum time trajectory $T_{1}$ of $C T$ is selected and collisions are checked as detailed in Section 3.4.2. If $T_{1}$ has a collision, it is taken out of $C T$ and the process returns to the step in Section 3.4.3 and is repeated until a solution is reached.

The proposed solution to the problem is an efficient trajectory without collisions which is an offspring trajectory resulting from the minimum time trajectory $T_{\min }$.

\section{Case Study}

The example shown in Figure 7 has been solved. The initial and final configurations are $C i(x 1, y 1)$ and $C f\left(x_{2}, y_{2}\right)$. 


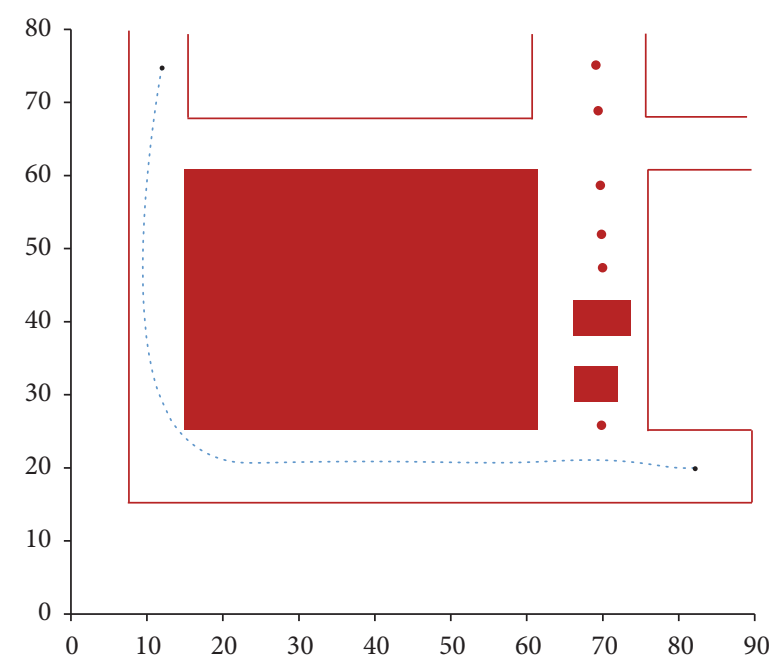

Figure 7: Initial and final car-like robot configuration.

The coefficient of adhesion between the ground and the tire may vary depending on several factors due to working conditions and time. These factors cover environmental conditions (such as wet, dry, or dirty road surfaces, moisture content, and temperature), technical characteristics of the tire, tire pressures, tire wear and maintenance standards, and so forth. The variations of the coefficient of adhesion have an important effect on the optimal trajectory time of the car-like autonomous robot, that is, the execution time of the robot task. Furthermore, they play a major role in how the robot performs its tasks appropriately, its productivity, and safety considerations.

This paper carries out an assessment of uncertainty based on the effects of the coefficient of adhesion regarding the optimal trajectory time by means of Monte Carlo simulations. Furthermore, the effects of the coefficient of adhesion over different variables are also analysed, which cover minimum trajectory time, maximum drive torque, maximum velocity, maximum braking torque, and consumed and dissipated energy. In addition, the computational time to generate an optimal trajectory is also provided.

4.1. Monte Carlo Simulations. The Monte Carlo simulations are based on the same case study and make use of the same car-like autonomous robot. 100 simulations have been run. All simulations share the same working conditions, initial and final configuration, and obstacles in the generated collisionfree trajectories. The only changed parameter is the coefficient of adherence. Values of the coefficient of adherence are sampled from a Gaussian statistical distribution with a mean $(m)$ of 0.25 and a standard deviation $(s)$ of $m / 3$, that is, 0.0833 . In this way, we reproduce the actual values of the coefficient of adherence which we find in real working conditions.

4.2. Results and Findings. Figure 8 shows the optimal trajectory time versus the coefficient of adherence for an ensemble of a hundred simulations and also its trend line. Results show that, as expected, lower minimum times are achieved for higher values of the coefficient of adherence due to the greater

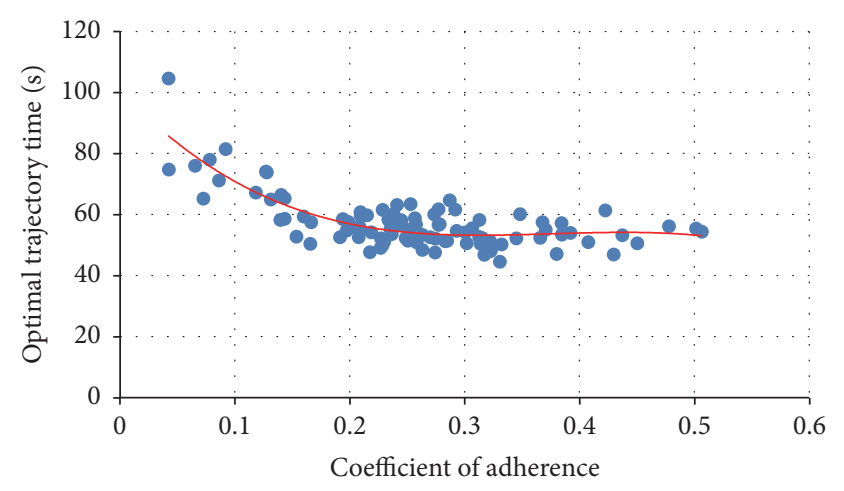

FIGURE 8: Optimal trajectory time (s) versus the coefficient of adherence for an ensemble of a hundred simulations (blue dotted points) and also its trend line (red line).

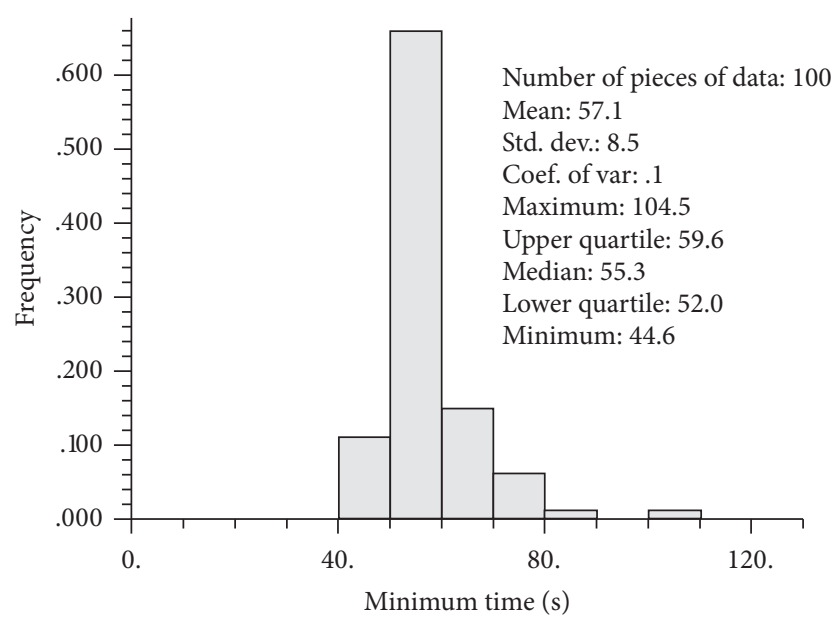

FIGURE 9: Frequency distribution and univariate statistics for the minimum trajectory time.

grip forces between tires and terrain. The minimum time is a discontinuous function due to numerical discrepancies in the coupling of the kinematic and dynamic model of the robot and the SQP method to solve the nonlinear optimization problem.

The frequency distribution and univariate statistics for the minimum trajectory time are depicted in Figure 9. The distribution has a mean of $57.1 \mathrm{~s}$ and a standard deviation of $8.5 \mathrm{~s}$, where the minimum trajectory time ranges from $0.04 s$ to $0.5 s$. These results show that the minimum trajectory time also follows a Gaussian distribution with a lower standard deviation in response to the Gaussian statistical distribution of the coefficient of friction. On the other hand, the results also show the importance of taking into account the uncertainty in the coefficient of adherence and the worth of the presented methodology. This is even more critical when considering that a robot task may be repeated constantly, for instance, in an assembly line. In this case, the minimum time has a great influence in production and economic considerations. The uncertainty assessment carried out may help in defining an efficient scheduling process of the robot's tasks. 


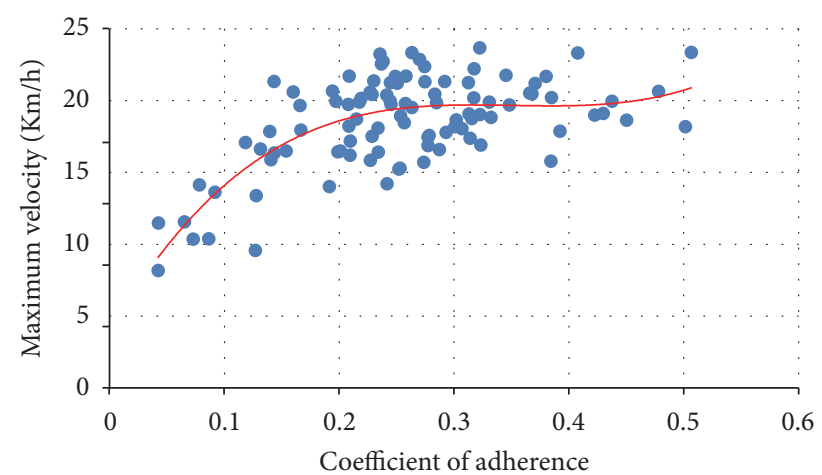

Figure 10: Maximum velocity $(\mathrm{km} / \mathrm{m})$ versus the coefficient of adherence for an ensemble of a hundred simulations (blue dotted points) and also its trend line (red line).

It means that we can use the Gaussian distribution of the coefficient of friction to predict, if necessary, the optimal trajectory times for the car-like robot to travel or do a task.

Figure 10 presents the maximum velocity versus the coefficient of adherence. It can be seen that higher velocities are achieved for greater grip forces, that is, for greater coefficient of adherence values. These results are in line with those obtained for the minimum trajectory time. Again, a wide dispersion in the velocity is obtained for the different simulations. The followed path will not always be the same and small radius of curvature limits the robot's speed. Also note that the velocities values stabilize from the mean value of the Gaussian distribution of the coefficient of adherence $(0.25)$. It is advisable to work with values of velocities as high as possible. Also bear in mind that velocities range from $8 \mathrm{~km} / \mathrm{h}$ to $24 \mathrm{~km} / \mathrm{h}$. The maximum value is also limited by the driving torque and the kinematics and dynamics characteristics of the car-like robot.

Figure 11 represents the maximum driving torque on the rear wheels versus the coefficient of adherence, which shows that the driving torque is limited by the value of the coefficient of adherence. With small values of the coefficient of adherence, the driving torque cannot be bigger than the friction force in tires to avoid slippage. The maximum values are also limited by the maximum torque provided by the engine and the followed path which can be very sinuous. Initially, the path is sinuous and the engine cannot give the maximum driving torque. Also, at higher velocities, the driving torque decreases.

The maximum braking torque in the front wheels also strongly depends on the coefficient of adherence (Figure 12). It is shown that the higher the grip force is the higher the braking torque obtained is.

Figure 13 shows the computational time for the different simulations. They present low computational cost for all simulations with a mean of $1.8 s$, very small value that shows the efficiency of the algorithm. It can also be seen that the trend line slightly decreases for higher values of the coefficient of adherence due to better behaviour of those values in the nonlinear optimization procedure to obtain minimum time trajectories.

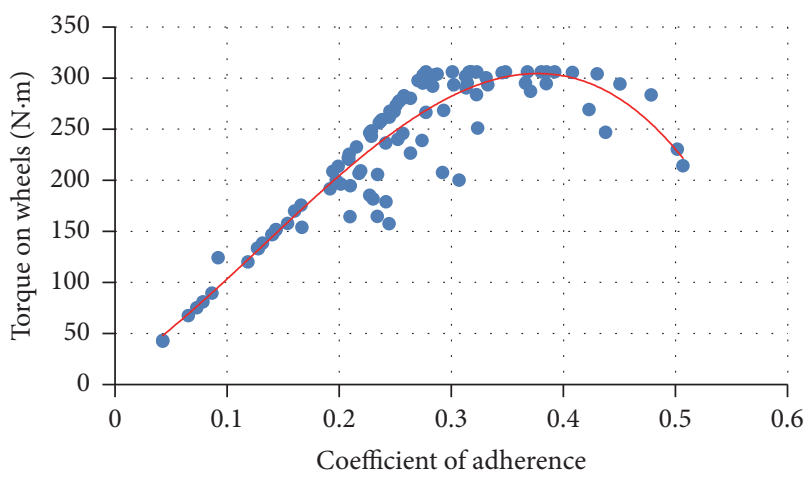

FIGURE 11: Maximum driving torque on rear wheels $(\mathrm{N} \cdot \mathrm{m})$ versus the coefficient of adherence for an ensemble of a hundred simulations (blue dotted points) and also its trend line (red line).

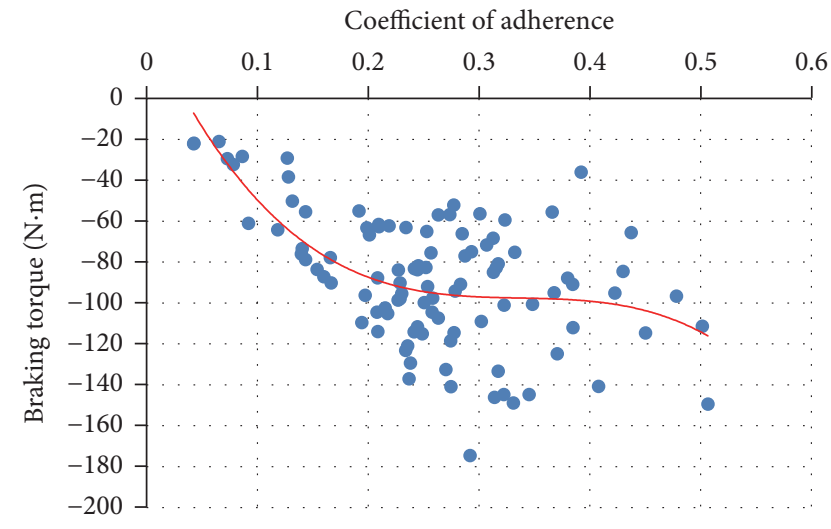

FIGURE 12: Maximum braking torque in the front wheels $(\mathrm{N} \cdot \mathrm{m})$ with regard to the coefficient of adherence for an ensemble of a hundred simulations (blue dotted points) and also its trend line (red line).

Finally, Figure 14 presents the energy consumed and dissipated for the autonomous robot for the different simulations. It shows that the energy balance is equal to the energy consumed minus the energy dissipated. It also shows that the energy dissipated increases with the coefficient of adherence, while the energy balance remains almost constant for all simulations, with a mean value of $6678(\mathrm{~J})$.

\section{Conclusions}

The simplified dynamic model of the robotic vehicle makes it possible to achieve safe, collision-free, feasible trajectories while fulfilling the dynamic capabilities of the robot, with moderate computation times.

Efficient trajectories are obtained for complex environments with scattered obstacles, always respecting the limitations of the robot dynamics.

The methodology allows determining all the important working variables in the calculation of an optimal robot trajectory (i.e., minimum trajectory time, maximum velocity, torque on wheels, braking torque, computational cost, consumed and dissipated energy, and energy balance). Furthermore, the methodology allows dealing with uncertain environments and a wide range of real case studies by 


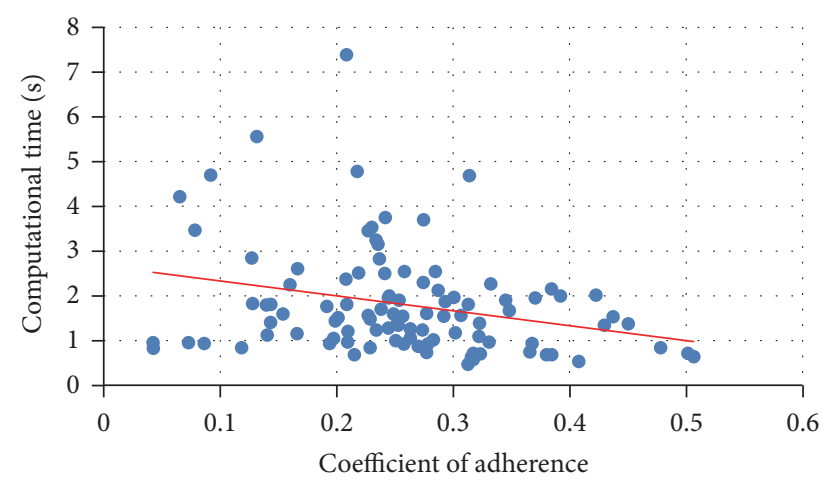

FIGURE 13: Computing time for the different simulations.

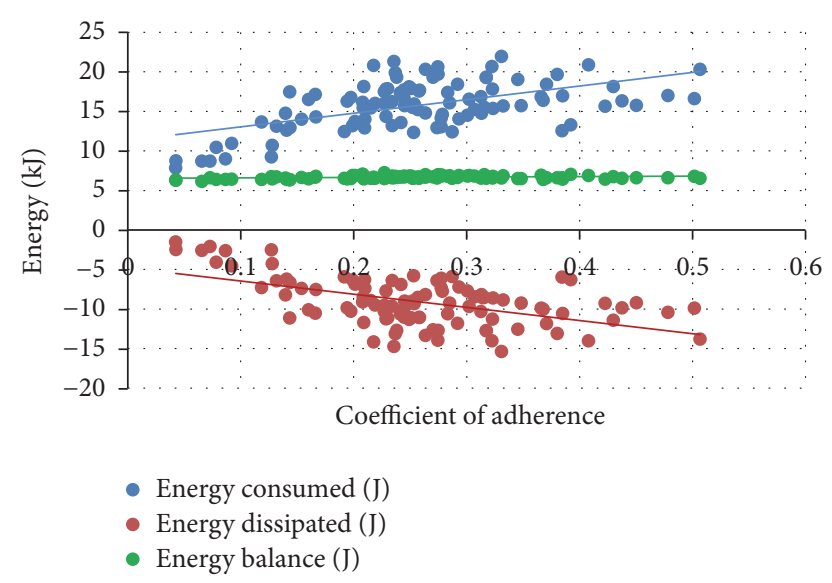

FIgURE 14: Consumed and dissipated energy for the autonomous robot and energy balance (J).

taking in consideration the kinematics and dynamics of the autonomous robot and providing optimal and collision-free trajectories.

From the graphs, it can be concluded that a relatively large coefficient of friction is good for most of the working parameters except for the consumed and dissipated energy. Because the energy is not recovered, high consumption is not recommended. Because we are using as objective function the time needed to perform the trajectory, this has an energetic cost.

Finally, based on this algorithm, a trajectory planner for robotic vehicles equipped with instrumentation to detect moving obstacles can be developed, since the computation times would allow trajectories to be recalculated according to the obstacles' motion. This is a topic that could be further developed in future work.

\section{Conflicts of Interest}

The authors declare that they have no conflicts of interest.

\section{Acknowledgments}

This work was supported by the Spanish Ministry of Economy and Competitiveness, which has funded the DPI2013-44227$\mathrm{R}$ project.

\section{References}

[1] D. Adminaite, R. Allsop, and G. Jost, "Ranking EU progress on road safety. 9th road safety performance index report," Tech. Rep., European Transport Safety Council (ETSC), 2015.

[2] R. Siegwart and R. Nourbakhsh, Introduction to Autonomous Mobile Robots, MIT press, 2004.

[3] J. Peng, W. Luo, W. Liu, W. Yu, and J. Wang, "A suboptimal and analytical solution to mobile robot trajectory generation amidst moving obstacles," Autonomous Robots, vol. 39, pp. 1-23, 2015.

[4] C. Katrakazas, M. Quddus, W. H. Chen, and L. Deka, "Real-time motion planning methods for autonomous on-road driving: State-of-the-art and future research directions," Transportation Research, vol. 60, pp. 416-442, 2015.

[5] J. Perez, J. Godoy, J. Villagni, and E. Onieva, "Trajectory generator for autonomous vehicles in urban environments," in Proceedings of the IEEE International Conference on Robotics and Automation (ICRA), 2013.

[6] K. Kawabata, L. Ma, J. Xue, and N. Zhemg, "A path generation for automated vehicle based on Bezier curve and via-points," in Proceedings of the EEE/ASME International Conference on Advanced Intelligent Mechatronics (AIM), 2013.

[7] M. Elbanhawi, M. Simic, and R. N. Jazar, "Continuous path smoothing for car-like robots using B-spline curves," Journal of Intelligent Robotic Systems, vol. 80, pp. 23-56, 2015.

[8] A. Broggi, P. Medici, P. Zani, A. Coati, and M. Panciroli, "Autonomous vehicles control in the VisLab intercontinental autonomous challenge," Annual Reviews in Control, vol. 36, no. 1, pp. 161-171, 2012.

[9] B. Nagy and A. Kelly, "Trajectory generation for car-like robots using cubic curvature polynomials," In Field and Service Robots, 2001.

[10] M. Wang, T. Ganjineh, and R. Rojas, "Action Annotated Trajectory Generation for Autonomous Maneuvers on Structured Road Networks," in Proceedings of the 5th International Conference on Automation, Robotics and Applications, 2011.

[11] D. Wang and F. Qi, "Trajectory planning for a four-wheelsteering vehicle," in Proceedings of the 2001 IEEE International Conference on Robotics \& Automation Seoul, 2001.

[12] J. H. Jeon, R. V. Cowlagi, S. C. Peters et al., "Optimal motion planning with the half-car dynamical model for autonomous high-speed driving," in Proceedings of the American Control Conference (ACC), 2013.

[13] Y. Cong, O. Sawodny, H. Chen, J. Zimmermann, and A. Lutz, "Motion planning for an autonomous vehicle driving on motorways by using flatness properties," in Proceedings of the 2010 IEEE International Conference on Control Applications part of 2010 IEEE Multi-Conference on Systems and Control, IEEE, Yokohama, Japan, September 2010.

[14] S. Staicu, "Dynamics equations of a mobile robot provided with caster wheel," Nonlinear Dynamics, vol. 58, no. 1-2, pp. 237-248, 2009.

[15] K. R. Simba, N. Uchiyama, and S. Sano, "Real-time obstacleavoidance motion planning for autonomous mobile robots," in the Australian Control Conference, 2014.

[16] K. R. Simba, N. Uchiyama, and S. Sano, "Real-time smooth trajectory generation for nonholonomic mobile robots using Bézier curves," Robotics and Computer-Integrated Manufacturing, vol. 41, pp. 31-42, 2016.

[17] B. Li and Z. Shao, "Simultaneous dynamic optimization: a trajectory planning method for nonholonomic car-like robots," Advances in Engineering Software, vol. 87, pp. 30-42, 2015. 
[18] P. Tokekar, N. Karnad, and V. Isler, "Energy-optimal trajectory planning for car-like robots," Autonomous Robots, vol. 37, pp. 279-300, 2014.

[19] F. Rubio, F. Valero, J. L. Sunyer, and A. Garrido, “The simultaneous algorithm and the best interpolation function for trajectory planning," Industrial Robot, vol. 37, no. 5, pp. 441-451, 2010.

[20] J. Cazalilla, M. Vallés, V. Mata, M. Díaz-Rodríguez, and A. Valera, "Adaptive control of a 3-DOF parallel manipulator considering payload handling and relevant parameter models," Robotics and Computer-Integrated Manufacturing, vol. 30, no. 5, pp. 468-477, 2014.

[21] X. Iriarte, J. Ros, V. Mata, and A. Plaza, "Multibody model reduction by parameter elimination," in Proceedings of the ECCOMAS Thematic Conference on Multibody Dynamics, 2015.

[22] R. Pastorino, E. Sanjurjo, A. Luaces, M. A. Naya, W. Desmet, and J. Cuadrado, "Validation of a Real-Time Multibody Model for an X-by-Wire Vehicle Prototype Through Field Testing," Journal of Computational and Nonlinear Dynamics, vol. 10, no. 3, Article ID 031006, 2015.

[23] J. L. Suñer, F. Valero, J. J. Ródenas, and A. Besa, Comparación Entre Procedimientos de Solución de La Interpolación Por Funciones Splines Para La Planificación de Trayectorias de Robots Industriales, 2007.

[24] F. Rubio, F. Valero, J. Sunyer, and V. Mata, "Direct step-by-step method for industrial robot path planning," Industrial Robot: An International Journal, vol. 36, no. 6, pp. 594-607, 2009. 


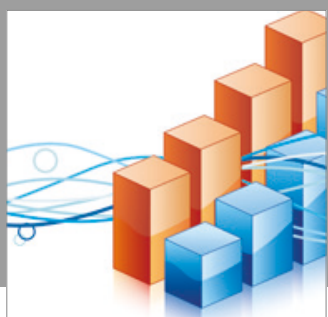

Advances in

Operations Research

vatersals

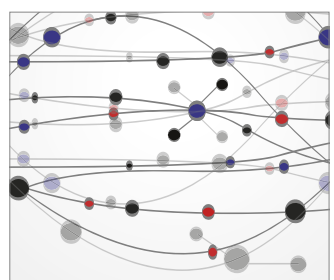

\section{The Scientific} World Journal
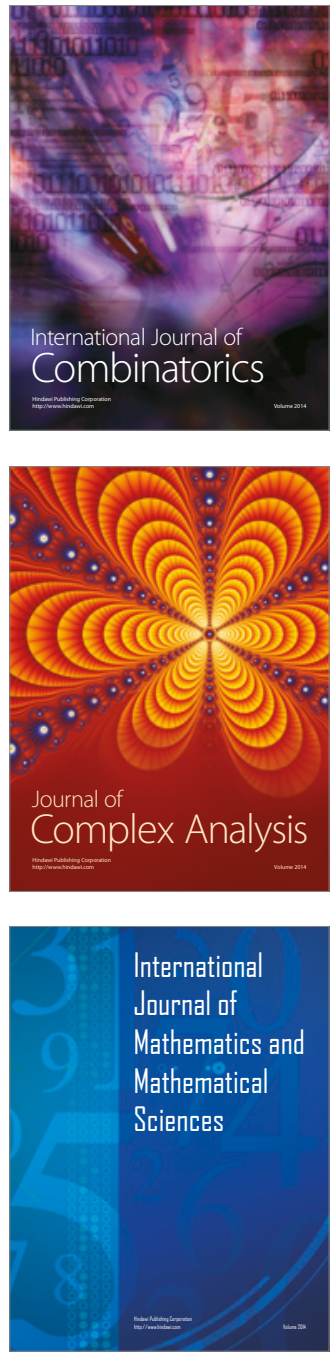
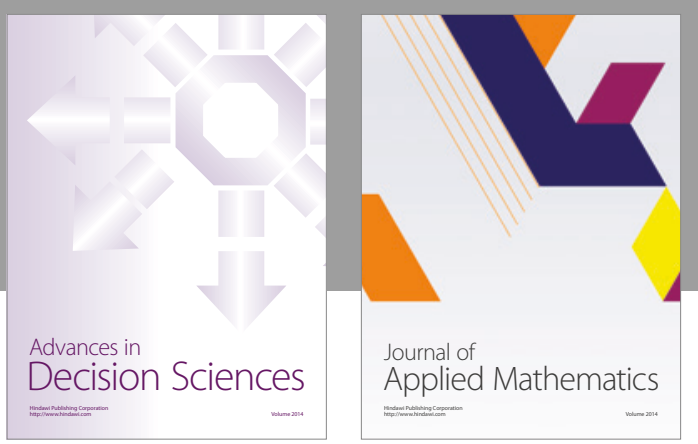

Algebra

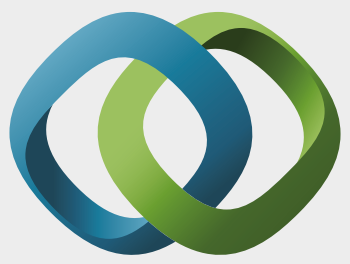

\section{Hindawi}

Submit your manuscripts at

https://www.hindawi.com
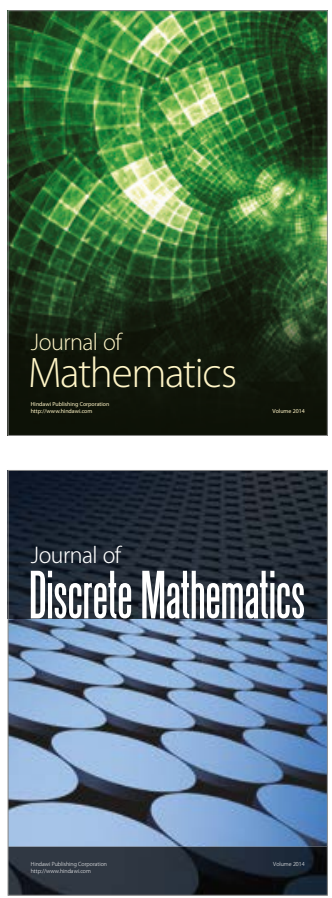

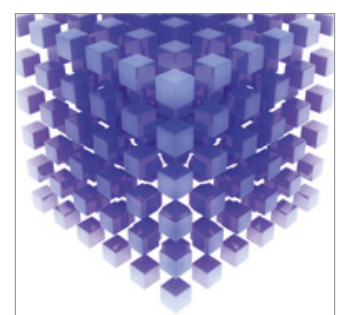

Mathematical Problems in Engineering
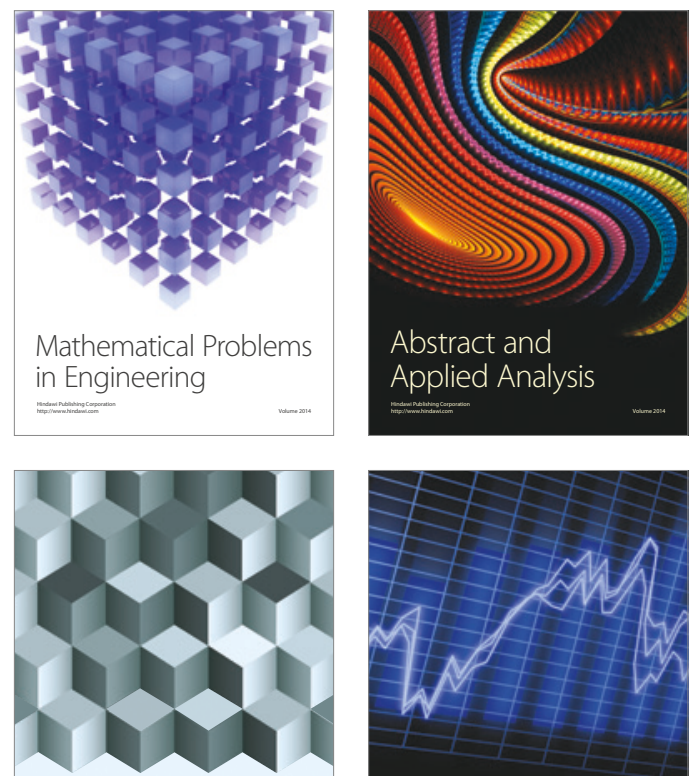

Journal of

Function Spaces

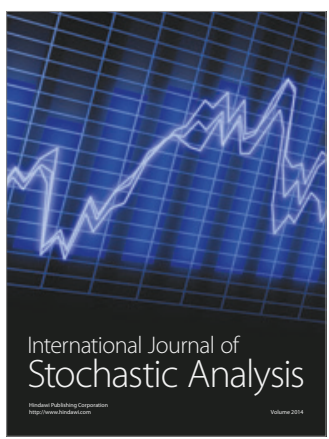

Probability and Statistics
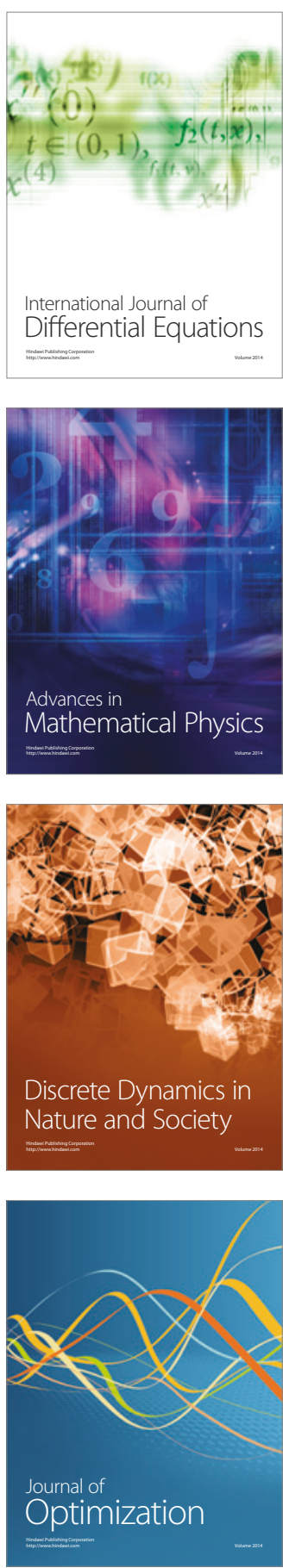